Comments Procedures for donors attention, registration, storage, transportation and milk collection from attention points to the Milk Bank should preserve the security and quality.

The comparison of average acidity according to the origin of milk, coul be an adequate advisor in order to promote better strategies to minimise the percentage of rejected milk.

\section{PO-0607a THE BENEFIT OF COMPUTER ASSISTED PRESCRIPTION OF PARENTERAL NUTRITION IN ELGANS}

II Stucin Gantar, ${ }^{1}$ A Pogorelc Erjavec, ${ }^{2} \mathrm{M}$ Mramor, ${ }^{3} \mathrm{~J}$ Zbontar. ${ }^{1}$ Department of Gynecology and Obtetrics, University Medical Centre, Ljubljana, Slovenia; ${ }^{2}$ Children Hospital, University Medical Centre, Ljubljana, Slovenia; ${ }^{3}$ Faculty of Computer Science and Informatics Ljubljana, University Ljubljana, Ljubljana, Slovenia

\subsection{6/archdischild-2014-307384.1247}

Background To minimise extrauterine growth restriction in ELGANs computer assisted prescription of parenteral nutrition (CAPPN) was introduced.

Aim To evaluate the effectiveness of CAPPN in growth improvement of ELGANs.

Patients and methods In this retrospective, observational designed study with a nonprobability, convenience sampling to obtain medical records, we compared 20 ELGANs in the study group after CAPPN to 20 ELGANs in the control group before CAPPN. Daily parenteral and enteral intake of macronutrients, calcium and phosphate in five sequetial time intervals of the first 28 days of life was calculated (day 1-3, 4-7, 2nd, 3rd, 4th week). Outcome measures were the lenght of PN, days to regain birth weight (BW), growth velocity, and weight and head circumpherence $(\mathrm{HC})<10$ th percentile on day 28. Numerical data were analysed by independent-samples t-test or by Mann-Whitney $U$ test, categorical data were analysed by chi-square.

Results The combined enteral and parenteral intake of the study group in all five sequential intervals after birth exceeded the intake of the control group. The lenght of $\mathrm{PN}$ and days to regain BW did not differ, however growth velocity $(14,5[3,7]$ vs 11.6 $[0,4] \mathrm{g} /$ day $(\mathrm{p}=0.03))$ and $\mathrm{HG}$ velocity $(0.9$ [0.3] vs $0.7[0.4]$ $\mathrm{cm} /$ day $(\mathrm{p}=0.03))$ were higher in the study group. Less growth retardation on day 28 was obtained (weight $2 / 20$ vs $9 / 20$ (p < $0.001)$; HC $3 / 20$ vs $13 / 20$ ( $<<0.001)$ ).

Conclusion In ELGANs delivery of nutrients and growth during the first month of life were significantly improved with CAPPN.

\section{PO-0607b METABOLOMIC DETERMINANTS OF NECROTIZING ENTEROCOLITIS IN PRETERM PIGLETS}

${ }^{1} \mathrm{~L}$ Call, ${ }^{1} \mathrm{~B}$ Stoll, ${ }^{1} \mathrm{~S}$ Garcia, ${ }^{1} \mathrm{C}$ Bauchart-Thevret, ${ }^{1} \mathrm{~J}$ Donnelly, ${ }^{2} \mathrm{~F}$ Sheikh, ${ }^{2} \mathrm{~A}$ Akinkuotu, ${ }^{2} \mathrm{O}$ Olutoye, ${ }^{3} \mathrm{~A}$ Wittke, ${ }^{1} \mathrm{D}$ Burrin. ${ }^{1}$ Pediatrics, Children's Nutrition Research Center/ Baylor College of Medicine, Houston, USA; ${ }^{2}$ Surgery, Texas Children's Hospital Division of Pediatric Surgery, Houston, USA; ${ }^{3}$ Nutrition, Mead Johnson Pediatric Nutrition Institute, Houston, USA

\subsection{6/archdischild-2014-307384.1248}

Background and aim Studies in premature infants and animals show that carbohydrate malabsorption and gut microbiota colonisation are key elements for triggering necrotizing enterocolitis (NEC). Our aim was to determine how dietary carbohydrate composition affects the metabolomic profile and whether unique metabolite signatures correlate with NEC incidence.

Methods Cecal contents and plasma were collected from a group of preterm pigs at birth and from three groups fed formula containing either lactose, corn syrup solids (CSS) or a 1:1 mixture of lactose:CSS (MIX) as the sole carbohydrate. We performed metabolomic analysis by LC/GC mass spectroscopy, clinical and histological NEC scoring, and distal ileum tissue expression of inflammatory markers.

Results Based on clinical and histological scores NEC incidence rates were $12 \%, 35 \%$, and $40 \%$ in the lactose, CSS and MIX groups, respectively. Ileum inflammatory markers (IL-8, IL-6, and IL1b) were highest in CSS vs. MIX and lactose groups and also correlated with NEC. Metabolomic analysis showed that lactose vs. CSS formula increased abundance of several cecal endocannabinoids. CSS and MIX formula increased plasma histamine, cecal and plasma lactate, beta-hydroxybutyrate, and butanediol, and decreased the abundance of several primary and secondary bile acids vs. lactose fed pigs.

Conclusions We conclude that lactose-based formula protects against inflammation and NEC and that this correlates with increased cecal levels of anti-inflammatory neurotransmitters and reduced levels of carbohydrate fermentation products and bile acids. This novel finding suggests that endocannabinoids, normally found in breast milk, may be produced endogenously and modulate inflammation in preterm neonates fed a lactose-based formula.

\section{PO-0607C ADMINISTRATION OF BIFIDOBACTERIUM BREVE AND LACTOBACILLUS SALIVARIUS, TWO STRAINS ISOLATED FROM HUMAN MILK, TO VERY LOW AND EXTREMELY LOW BIRTH WEIGHT PRETERM INFANTS:A PILOT STUDY}

${ }^{1}$ E Escribano, ${ }^{2}$ L Moles, ${ }^{2} \mathrm{~J}$ De Andres, ${ }^{3}$ E Jimenez, ${ }^{3}$ I Espinosa-Martos, ${ }^{2} \mathrm{JM}$ Rodriguez, ${ }^{1} \mathrm{M}$ Saenz De Pipaon. 'Servicio de Neonatología, Hospital Universitario La Paz, Madrid, Spain; ${ }^{2}$ Bromatología Nutrición y Tecnología de Los Alimentos, Universidad Complutense de Madrid, Madrid, Spain; ${ }^{3}$ Probisearch S. L., PTM, Madrid, Spain

\subsection{6/archdischild-2014-307384.1249}

Preterm infant gut has been described as immature and colonised by an aberrant microbiota.

Objectives Elucidate if administration of two probiotic strains isolated from human milk to preterm infants led to their presence in faeces. Secondarily, evolution of immunological compounds in blood and faecal samples was also assessed.

Materials and methods Inclusion criteria: Birth weight $<1,300$ g, gestational age $<29$ weeks. Preterms received two daily doses $\left(\sim 10^{9} \mathrm{CFU}\right)$ of a mixture of B. breve PS12929 and L. salivarius PS12934 after meconiorrhexis. Meconium samples were collected prior to and faecal and blood samples were collected weekly for up to 28 days. Faecal bacterial growth was detected by culture-dependent techniques. Cytokines, chemokines, growth factors and immunoglobulins were determined by multiplex technologies. The statistical analysis was performed using R2.15.3.

Result and discussion Supplementation of five with this probiotics was effective in enhancing the levels of L. salivarius PS12934 that could be isolated from day 7 of intervention and its presence remained constant throughout the study; B. breve PS12929 was be detected later, after day 14 , but had an increasing presence in the faecal samples. IL-4, IL-10 and IL-13 concentrations, related to anti-inflammatory processes, and IL- 8 and MCP-1 were similar to those values previously reported for 'late-preterms' at 7 days of life, this may reflect the immumodulatory activity of the probiotic strains on this population. It demonstrated an increase in IgA since day 7. A reduction of 
calprotectin in faeces was observed throughout treatment. Globally, probiotic supplementation exerted a strong influence on gut colonisation.

\section{Neonatal Others}

\section{PO-0608 PAIN EVALUATION IN THE PRETERM}

${ }^{1} \mathrm{M}$ Ahmed, 'DN Sobithadevi, 'S Mostafa, ${ }^{1} \mathrm{P}$ Pore, ${ }^{2} \mathrm{~J}$ Maiden, ${ }^{2} \mathrm{~S}$ Boswell, ${ }^{1} \mathrm{M}$ Molnar, ${ }^{3} \mathrm{~T}$ Reynolds. ${ }^{1}$ Paediatrics, Burton Hospitals NHS Foundation Trust, Burton on Trent, UK; ${ }^{2}$ Research and Development, Burton Hospitals NHS Foundation Trust, Burton on Trent, UK; ${ }^{3}$ Clinical Chemistry, Burton Hospitals NHS Foundation Trust, Burton on Trent, UK

\subsection{6/archdischild-2014-307384.1250}

Background Assessment of pain is a challenge in neonatal setting. Visual, behavioural and physiological pain scales are not always reliable in premature infants. Few studies with limited sample size have been published on the reliability and efficacy of Skin Conductance Algesimeter (SCA) in monitoring pain in infants and children.

Aim To identify the clinical usefulness of SCA as a reliable and valid measure of pain intensity and stress response in preterm infants.

Methods Parents of all preterm infants admitted to the neonatal unit were invited to participate in the study. The usefulness of SCA was compared with simultaneous measurement of 'Premature Infant Pain Profile' (PIPP) and 'Face, Legs, Activity, Cry and Consolability (FLACC) scores during invasive and/or painful procedures.

Results 85 measurements were recorded. PIPP and FLACC scores started low, increased during the procedure and decreased afterwards. For all SCA measurements, there was an increase in score pre-pro and a decrease in score pro-post. However, the standard deviation for variations was wider for some measurements than for others. Paired t-test comparing Delta pre-pro with Delta pro-post for all measurements \{PIPP, FLACC, Area (small) and Peaks/sec $\}$ individually showed statistically significant differences $(p<0.05)$. For Area (small), there was no significant correlation between SCA data and PIPP/FLACC scores.

Conclusions SCA, PIPP and FLACC scores increased during the painful procedures. Although the SCA, PIPP and FLACC data is mathematically correlated, at a clinical level, the correlation is too imprecise to use the SCA to predict or measure behavioural responses to noxious stimuli in neonates.

\section{PO-0609 DISSEMINATED RENAL VEIN THROMBOSIS MAY BEGIN IN UTERO}

${ }^{1} \mathrm{~S}$ Aktas, ${ }^{1} \mathrm{~S}$ Unal, ${ }^{1} \mathrm{C}$ Turkyilmaz, ${ }^{2} \mathrm{C}$ Damar, ${ }^{2 B}$ Derinkuyu, ${ }^{2} \mathrm{O}$ Boyunaga, ${ }^{3} \mathrm{E}$ Sal, ${ }^{3} \mathrm{Z}$ Kaya, ${ }^{4} Y$ Ozdemir, ${ }^{4}$ SB Ezgu. ${ }^{1}$ Pediatrics Newborn Medicine, Gazi University School of Medicine, Ankara, Turkey; ${ }^{2}$ Pediatric Radiology, Gazi University School of Medicine, Ankara, Turkey; ${ }^{3}$ Pediatric Hematology, Gazi University School of Medicine, Ankara, Turkey; ${ }^{4}$ Pediatric Nephrology, Gazi University School of Medicine, Ankara, Turkey

10.1136/archdischild-2014-307384.1251

Introduction Renal vein thrombosis (RVT) in neonates is a rare condition that carries low mortality but high morbidity. Aetiology isn't fully understood; predisposing factors are dehydratation, sepsis, asphyxia, polycythemia, maternal diabetes, traumatic delivery, congenital renal vein defects, umblical catheterisation, prothrombotic conditions.
Case presentation 36 week baby was born by C/S to 32 yearold, gravida 2 mother. The only prenatal risk was gestational diabetes. She was born early because of fetal distress. Evaluation of the infant for jaundice revealed left flank mass and edematous left leg in the second day of life. No effusion was detected in the joints of hip and knee. Lower extremity doppler USG was normal. Abdominal USG showed enlarged left kidney. Doppler USG showed thrombus in the inferior venae cavae, extending to left renal vein, main iliac veins, right external iliac vein. Right renal vein drained to retroperitoneal collaterals. Abdominal tomography confirmed USG. Retrospectively antenatal history revealed enlarged left kidney determined by USG performed right before birth. Based on retroperitoneal collaterals and prenatal USG we think RVT probably began in utero. There was no evidence of sagittal sinus thrombosis. The neonate was treated with LMWH. The results of clotting studies of mother were normal; heterozygot mutation of factor V Leiden and MTHFR gene were found in the baby. Follow-up renal scan at 3 months documented a non-functioning left kidney.

Conclusion In neonatal period; when renal vein thrombosis and disseminated thrombosis is detected in the absence of other risk factors, prothrombotic conditions should be searched.

\section{P0-0610 NEONATAL READMISSION FOR INDIRECT HYPERBILIRUBINEMIA IN AL AHSA, SAUDI ARABIA}

${ }^{1}$ A Al Omran, ${ }^{2}$ Z Al Salam, ${ }^{3}$ S Al-Abdi. ${ }^{1}$ Pediatrics, Almana General Hospital, Al Hafoof Wal Mubarraz, Saudi Arabia; ${ }^{2}$ Pediatrics, AlRiyadh Military Hospital, Riyadh, Saudi Arabia; ${ }^{3}$ Pediatrics, King Abdulaziz Hospital for Nathional Guard, Al-Ahsa, Saudi Arabia

\subsection{6/archdischild-2014-307384.1252}

Background Causes and severity of neonatal indirect hyperbilirubinemia $(\mathrm{IH})$ vary geographically. Understanding local differences may assist in prevention of complications.

Objective To study the causes and severity of $\mathrm{IH}$ in readmitted neonates in $\mathrm{Al}$ Ahsa district.

Methods Records of all neonates readmitted for IH to Almana General Hospital at Al Ahsa between 2009 and 2013 were reviewed. Data included: maternal and neonatal characteristics, laboratory results, associated causes of $\mathrm{IH}$ and interventions.

Results 323 neonates were readmitted for IH (gestational age: $38 \pm 1.8$ weeks, birth weight: $3023 \pm 513 \mathrm{~g}$ and males: 66\%). The age and weight at readmission were $125 \pm 770 \mathrm{~h}$ and 2923 $\pm 508 \mathrm{~g}$ respectively. History of previous jaundiced sibling was documented in $15 \%$. The most common cause for IH was G6 PD deficiency (39.9\%), followed by ABO incompatibility (12\%) and breast milk jaundice (11\%). The cause could not be determined in $31 \%$.

Based on 2004-AAP phototherapy guideline, 30\% infants were categorised as at lower risk, 58\% at medium and $14 \%$ at higher risk. The highest serum bilirubin during readmission was $322 \pm 59 \mu \mathrm{mol} / \mathrm{L}$ (range:198-686 $\mu \mathrm{mol} / \mathrm{L}$ ). All infants received phototherapy, and 2 required blood exchange transfusions. One case of kernicterus was documented (highest serum bilirubin: $686 \mu \mathrm{mol} / \mathrm{L}$ ) with no mortality. The median length of hospital stay was 2 days. $25(7.7 \%)$ required more than one admission.

Conclusions In $\mathrm{Al}$ Ahsa, the commonest cause of neonatal indirect hyperbilirubinemia is G6 PD deficiency. Screening for G6 PD deficiency and close postnatal follow up are recommended. 